\title{
BMJ Open Adherence to oral anticoagulants in patients with atrial fibrillation - a population-based retrospective cohort study linking health information systems in the Valencia region, Spain: a study protocol
}

\author{
G Sanfélix-Gimeno, ${ }^{1,2} \mathrm{C}$ L Rodríguez-Bernal, ${ }^{1,2}$ I Hurtado, ${ }^{1,2} \mathrm{C}$ Baixáuli-Pérez, ${ }^{1,2}$ \\ J Librero, ${ }^{1,2}$ S Peiró ${ }^{1,2}$
}

To cite: Sanfélix-Gimeno G, Rodríguez-Bernal CL, Hurtado I, et al. Adherence to oral anticoagulants in patients with atrial fibrillation -a population-based retrospective cohort study linking health information systems in the Valencia region, Spain: a study protocol. BMJ Open 2015;5: e007613. doi:10.1136/ bmjopen-2015-007613

- Prepublication history for this paper is available online. To view these files please visit the journal online (http://dx.doi.org/10.1136/ bmjopen-2015-007613).

Received 8 January 2015 Revised 24 June 2015 Accepted 17 August 2015

CrossMark

\begin{abstract}
${ }^{1}$ Health Services Research Unit, Fundación para el Fomento de la Investigación Sanitaria y Biomédica de la Comunidad Valenciana (FISABIO), Valencia, Spain ${ }^{2}$ Red de Investigación en Servicios de Salud en Enfermedades Crónicas (REDISSEC), Valencia, Spain
\end{abstract}

Correspondence to Dr G Sanfélix-Gimeno; sanfelix_gab@gva.es

\section{ABSTRACT}

Introduction: Adherence to oral anticoagulation (OAC) treatment, vitamin $\mathrm{K}$ antagonists or new oral anticoagulants, is an essential element for effectiveness. Information on adherence to OAC in atrial fibrillation (AF) and the impact of adherence on clinical outcomes using real-world data barely exists. We aim to describe the patterns of adherence to OAC over time in patients with $\mathrm{AF}$, estimate the associated factors and their impact on clinical events, and assess the same issues with conventional measures of primary and secondary adherence - proportion of days covered (PDC) and persistence-in routine clinical practice.

Methods and analysis: This is a population-based retrospective cohort study including all patients with $\mathrm{AF}$ treated with OAC from 2010 to date in Valencia, Spain; data will be obtained from diverse electronic records of the Valencia Health Agency. Primary outcome measure: adherence trajectories. Secondary outcomes: (1) primary non-adherence; (2) secondary adherence: (a) PDC, (b) persistence. Clinical outcomes: hospitalisation for haemorrhagic or thromboembolic events and death during follow-up. Analysis: (1) description of baseline characteristics, adherence patterns (trajectory models or latent class growth analysis models) and conventional adherence measures; (2) logistic or Cox multivariate regression models, to assess the associations between adherence measures and the covariates, and logistic multinomial regression models, to identify characteristics associated with each trajectory; (3) Cox proportional hazard models, to assess the relationship between adherence and clinical outcomes, with propensity score adjustment applied to further control for potential confounders; (4) to estimate the importance of different healthcare levels in the variations of adherence, logistic or Cox multilevel regression models.

Ethics and dissemination: This study has been approved by the corresponding Clinical Research Ethics Committee. We plan to disseminate the project's findings through peer-reviewed publications and

\section{Strengths and limitations of this study}

- This is a population-based study using realworld data to assess adherence to oral anticoagulation (OACs) and its association with effectiveness and safety outcomes.

- The study considers information on what physicians prescribe, and also on what patients fill from the pharmacy.

- The study considers multiple indicators of adherence, including group-based trajectory patterns, taking into account the dynamic nature of adherence.

- The observational nature of the study might lead to selection bias and confounding. Propensity scores will be used to address this issue.

- Results on use and adherence to OACs might not be generalisable to other settings.

presentations at relevant health conferences. Policy reports will also be prepared in order to promote the translation of our findings into policy and clinical practice.

\section{INTRODUCTION}

Atrial fibrillation (AF), the most common sustained arrhythmia, favours embolic stroke, which is one of the leading causes of cerebrovascular morbidity, neurological disability, quality of life loss and death. ${ }^{12}$ Prevalence in population-based studies in industrialised countries is 6.6 men and 3.9 women for every 1000 people of the respective gender, with a strong age gradient. ${ }^{3}$

Several randomised clinical trials (RCTs) have shown that preventive treatment with 
vitamin $\mathrm{K}$ antagonists (VKA) such as warfarin is highly effective, decreasing the incidence of cardioembolic stroke in patients with AF by approximately two-thirds, and thus reducing deaths and improving quality of life. ${ }^{4-8}$ Based on this evidence, low-intensity VKA therapy to maintain the international normalised ratio (INR) between 2.0 and 3.0 has, for many years, been the standard treatment for patients with $\mathrm{AF}$ at the highest risk of a stroke. $^{9} 10$

In recent years, however, new (non-VKA) oral anticoagulants (NOACs), such as dabigatran, rivaroxaban or apixaban, have been marketed. Their respective pivotal phase 3 trials reported similar or better thromboembolic event rates than warfarin and rates of haemorrhagic events similar to or less than those of warfarin. ${ }^{11-13}$ Several systematic reviews and meta-analyses confirm these results. ${ }^{14-19}$ Although some characteristics may favour one NOAC over another, direct comparisons are not available, and comparative effectiveness and safety remain unsatisfactorily tested. ${ }^{20}$ Several indirect comparisons have been performed showing conflicting results, ${ }^{21-32}$ and the validity of the conclusions from these studies is hindered by multiple methodological problems. ${ }^{33}$

The use of VKAs is associated with an increased risk of bleeding, regular blood monitoring, and drug-drug and drug-food interactions, and often imposes lifestyle changes. These factors lead to non-adherence, discontinuation of treatment and difficulties maintaining an optimal INR. ${ }^{34}$ Non-adherence and discontinuation of anticoagulant therapy leads to increased ischaemic stroke risk and contributes to suboptimal outcomes of the anticoagulant treatment. ${ }^{35-37}$ Owing to the scarcity of interactions, predictable effects with fixed dosages and the lack of need for INR monitoring, NOACs have some advantages over VKAs regarding comfort and convenience. However, they also have significant limitations, such as the unavailability of tests for monitoring their anticoagulant effectiveness, the absence of antidotes to reverse their effect, their renal clearance, some potential adverse effects and their higher cost (the cost per day of treatment with NOACs far outweighs that of VKAs, including monitoring costs). Furthermore, whether or not the advantages of NOACs may also translate into increased adherence and persistence is unclear. In fact, there is rising concern regarding poor adherence with these newer anticoagulant agents. ${ }^{38-40}$ In RCT studies, NOACs exhibit a persistence similar to that of warfarin, with only marginal differences. ${ }^{41}$ In the NOAC pivotal trials, permanent discontinuation stood between $21 \%$ and $25 \%,{ }^{11-13}$ figures that were not significantly different from their controls with warfarin (varying between $17 \%$ and $27 \%$ ), with median or mean follow-up ranging between 1.8 and 2 years. In real-world studies, and as expected since the conditions in trials are not generalisable to routine clinical practice, the warfarin persistence rates are lower than in clinical trials. ${ }^{42-51}$ Regarding NOACs, real-world data on persistence and adherence are sparse and heterogeneous, ${ }^{50}{ }^{52-54}$ and their association with effectiveness and safety outcomes has not been comprehensively examined in clinical practice; this association has been examined even less in settings such as Spain, where data may diverge considerably from those in the countries that participated in the trials. This is a relevant issue because patient characteristics, health system organisation and sociocultural contexts are significant determinants of adherence, and generalising drug adherence or persistence figures from one country to another may be an unreliable extrapolation. In the context of the Spanish National Health Service, information on adherence to anticoagulation therapy in $\mathrm{AF}$ and the impact of adherence on clinical outcomes barely exists.

At present, and although many factors have been shown to be strongly associated with discontinuation or non-adherence, ${ }^{55}$ our ability to distinguish or characterise non-adherent patients is poor. Additionally, adherence has been assessed in diverse ways, many of which are not standardised or replicable (eg, some studies confuse physician non-adherence to prescription guidelines with patient non-adherence to prescribed treatments, or use very different criteria for defining non-adherent patients, or mix naïve-drug cohorts with experienced-drug cohorts). Furthermore, not all studies use the same definitions regarding clinical outcomes (eg, some studies consider that the patient has discontinued treatment when switching from one anticoagulant to another).

Currently, the information available in electronic medical records enables the construction of observational cohorts for measuring adherence and persistence and its predictors, and for the assessment of their impact on clinical outcomes. ${ }^{56}{ }^{57}$ However, those studies based on electronic data sets usually share three important limitations: (1) they are based on limited groupssuch as those affiliated to a particular insurance company or a pharmaceutical benefit scheme-which are not necessarily representative of the general population, (2) they use pharmacy claims data with no information about physician prescription and, therefore, they combine physicians' failure to prescribe with patients' failure to refill the medication, and (3) they classify patients into groups of adherence using single indicators, overlooking the dynamic nature of non-adherence over time.

The use of advanced electronic prescription systems may be useful for addressing some of these drawbacks. The Valencia Health Agency (VHA), the public service responsible for healthcare in the Autonomous Community of Valencia (Spain), works under a scheme of universal coverage and tax-based funding. An extensive network of hospitals and primary healthcare centres are operated by the VHA, sharing an electronic medical record with an advanced electronic prescription system that includes the traceability of the prescription (from prescription by the doctor to the dispensing of the drug 
in pharmacies and subsequent claims to the VHA). These characteristics improve the representativeness of the population studied, and help differentiate between gaps in non-adherence owing to physicians' failure to prescribe from those related to the patients' failure to refill the medication.

Regarding the fact that adherence changes over time and that this aspect has been neglected in most existing studies, new methodological approaches such as groupbased trajectory models (GBTMs), a type of latent class analysis that incorporates information on the dynamic nature of adherence for its assessment, may also add some insights into the study of medication nonadherence. ${ }^{58}$ These alternative models estimate the change over time in an endpoint that is measured repeatedly, facilitating the qualitative identification of different sequences that define underlying subpopulations. GBTM is a person-centred approach (as is cluster analysis) focused on the relationships among individuals. $^{59}$ The most important outputs of the GBTM are the classification of patients into different trajectories over time (eg, nearly-always adherent patients, early gap in adherence after discharge with later recovery, brief gaps in medication use or occasional users, slow decline adherence, fast decline) and the description of such trajectories through easily interpretable graphics.

Although GBTMs have been widely used in some areas of medical and sociological research, and have an important potential to classify patients according to their long-term adherence, ${ }^{57}$ their use in studies of medication adherence is scarce ${ }^{57} 60-64$ Therefore, the availability of the aforementioned population-based electronic data sets and the use of new methodological approaches offer a great opportunity for assessing adherence to oral anticoagulation in a more comprehensive manner.

In summary, in spite of great efforts made in the scientific community and professional societies through clear guideline recommendations and quality of care policies based on solid evidence, underprescribing and low adherence to anticoagulation therapies remain a significant challenge in the management of patients suffering from AF, either because physicians fail to prescribe these medications and/or because patients fail to obtain and/ or use them. There are relatively few studies reporting the conventional measures of adherence (primary, persistence, percentage of days covered, etc) to VKA, and even fewer to NOAC, and none of them classify patients according to their adherence behaviour over time. Identifying adherence patterns, their associated factors and their relationship with clinical outcomes, would improve the ability of medical organisations to advance and focus more precisely on policies and interventions to improve their management.

\section{Objectives}

The main objective of the study is to describe the adherence trajectories (trajectory models or latent class growth analysis models) for oral anticoagulants, VKAs and NOACs, in patients with AF, and to estimate the associated factors and their impact on clinical events. We further aim to assess the same issues with the conventional measures of primary and secondary adherence (PDC and persistence) in routine clinical practice in a population-based setting.

The specific study objectives are: (1) to describe the trajectories or patterns of adherence oral anticoagulants in patients with $\mathrm{AF}$ in the Valencia region who have been treated with these drugs during the period 2010 present; (2) to estimate the likelihood of patients belonging to different trajectories and to identify the characteristics of the patients associated with each trajectory of adherence; (3) to estimate primary and secondary (proportion of days covered (PDC) and persistence) adherence to oral anticoagulants; (4) to identify factors associated with the conventional measures of nonadherence to oral anticoagulants, with reference to patient characteristics as well as those of the drugs themselves, the professionals and health organisations; (5) to estimate the impact on clinical outcomes of nonadherence (defined by descending patterns or trajectories of adherence, and by conventional measures of adherence).

\section{PATIENTS AND METHODS}

\section{Study design}

This is a population-based retrospective cohort study of all patients with $\mathrm{AF}$ on oral anticoagulant therapy from 2010 to date in the Valencia region. Some analyses will be carried out differentiating between naive patients (starting anticoagulant treatment for the first time in the period) and experienced patients (previously treated with anticoagulants).

\section{Population and setting}

The study will take place in the Valencia region and, specifically, in the population covered by the VHA, the public health system covering about $97 \%$ of the population of the region's population ( $\approx 5$ million inhabitants). All patients with AF (diagnosis code of International Classification of Diseases, Ninth Revision, Clinical Modification (ICD-9-CM) 427.31) under treatment with oral anticoagulants (warfarin, acenocoumarol, dabigatran, rivaroxaban, apixaban) since 2010 will be included. The time window will extend from 2009 to date to define the baseline characteristics of the population. We will define the naive population as those patients without anticoagulant treatment in the 12 months preceding the first prescription. People without pharmaceutical/health coverage by VHA, mainly some government employees whose prescriptions are reimbursed by civil service insurance mutualities, and thus not included in the pharmacy databases of the VHA, and patients not registered in the municipal census (non-residents or temporary residents), or who left the 
region or who were disenrolled from VHA coverage for other causes, were excluded because of limitations on follow-up.

\section{Data sources}

All necessary information will be obtained from the electronic information systems of the VHA (see figure 1): (1) The Population Information System (SIP) provides an identification number for each person under VHA coverage, and registers some demographic characteristics, and dates and causes of VHA discharge, including death. (2) The Minimum Basic Dataset (MBDS) at hospital discharge is a synopsis of clinical and administrative information on all hospital discharges, including diagnoses and procedures (all electronic health systems in the VHA use the ICD-9-CM). (3) The electronic medical record for ambulatory care, ABUCASIS, is available in all primary healthcare centres and other ambulatory settings. It has all the information on patients regarding diagnoses, their personal and family medical history, laboratory results, lifestyle, etc. (4) The pharmaceutical module, called GAIA (prescription information system), part of ABUCASIS, includes information about both physician prescriptions and dispensations from pharmacy claims. (5) The Corporate Resource Catalogue (CRC) provides information about the geographical and functional organisation of VHA, its health centres, health services provided and professionals in healthcare. All the information in these systems can be linked at an individual level through the SIP number.

\section{Outcome measures (main and secondary endpoints) Main outcome measure}

The primary outcome measure will be the patterns or trajectories of adherence to the different anticoagulants over the time of follow-up. By calculating the number of days covered by dispensed medication, monthly adherence will be ascertained from the time of the first prescription (defining being adherent as having $\geq 24$ days covered out of 30 ( $\geq 80 \%$ of the time covered)), obtaining a repeated dichotomous measure for each month of follow-up and patient. Patterns or adherence trajectories will be identified using group-based trajectory modelling, patients being classified according to their most likely pattern of adherence (ie, the trajectory that the patient has the highest probability of belonging to).

\section{Secondary outcome measures}

The following will be used as secondary measures (see figure 2): (1) Primary non-adherence, defined as not

Figure 1 Data sources and linkage between databases. A\&ED, accident and emergency department; CRC, Corporate Resource Catalogue; ICD-9-CM, International Classification of Diseases, Ninth Revision, Clinical Modification; MBDS, Minimum Basic Dataset; SIA, Ambulatory Information System. 


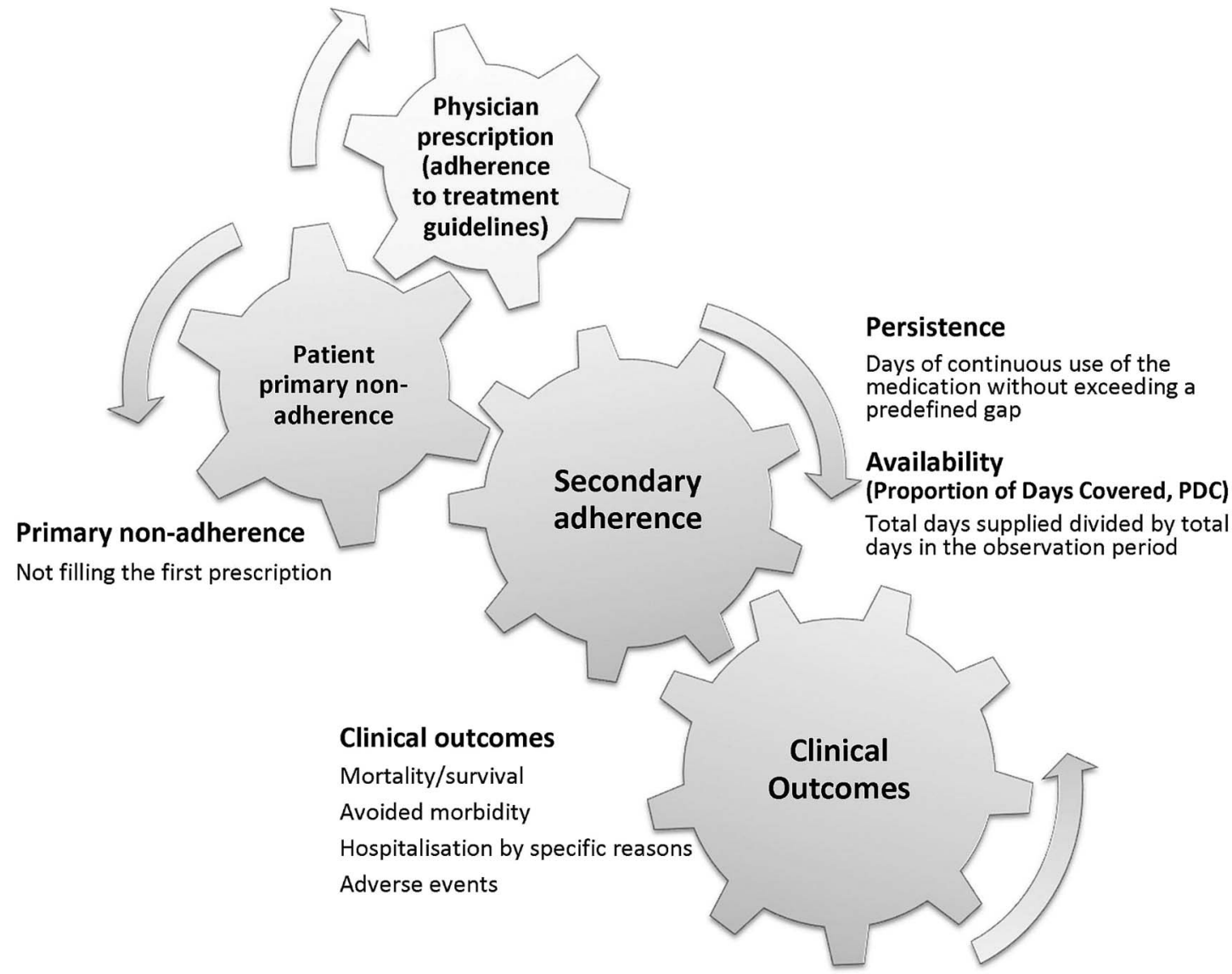

Figure 2 Secondary outcomes (measures of adherence) and clinical outcomes to be considered in the study.

filling the first prescription of a specific anticoagulant from the pharmacy. ${ }^{6566}$ The first prescription is defined as the first prescription identified in the electronic clinical record in the study period. (2) Secondary adherence, as assessed by: (a) availability, measured by the proportion of days covered (PDC) by medication. PDC is a widely used measure of adherence calculated by dividing the number of days of medication dispensed by the number of days of the observed follow-up period. This measure is very similar to the medication possession ratio, with the difference that the PDC is capped at $100 \%$ in case the days of medication dispensed exceed those of the follow-up period. ${ }^{67}{ }^{68}$ Days with available medication during the follow-up period will be estimated through the dose regimen defined by the physician and the number of pills per package (eg, for a regimen of one pill every $12 \mathrm{~h}$ and packages of 30 tablets, each dispensation will entail 15 days of medication available). We will allow a maximum of accumulated days supply (stockpiling) of 180 days. To classify patients as adherent, the widely accepted cut-off point of $80 \%$ will be used (alternative cut-off points will be tested in sensitivity analyses); and (b) persistence, defined as the duration of continuous use of the medication after the index prescription. The treatment will be considered discontinued if a patient does not fill the medication from the pharmacy after grace periods of 30 and 60 days, once the period with available medication from the previous dispensation is over. ${ }^{67}{ }^{69}$ In the sensitivity analysis, the impact of using shorter and longer grace periods will be estimated.

\section{Clinical outcomes}

We seek to include the following clinical outcomes (see figure 2): hospitalisation for thromboembolic events, bleeding events and death from any cause during the follow-up period (2010-to date). Such admissions will be identified through the corresponding ICD-9-CM codes in the MBDS, while the information about mortality (from any cause) will be obtained from the SIP.

\section{Covariates}

Covariates related to prescription, patients and level of healthcare system will be selected from the different electronic databases (see figure 1): (1) prescription-related variables: generic name, price, strength, dosing schedule/regimen, electronic dispensing, reduced co-payment ( $10 \%$ of the cost with a ceiling of $€ 4.13(\approx £ 3.27)$ per package for some long-term treatments); (2) patientrelated variables: date of birth, sex, co-payment level, comorbidity (eg, stroke, heart failure, ischaemic heart disease, valvular heart disease, arrhythmias, thyroid disease, diabetes, sleep apnoea, chronic obstructive pulmonary disease, chronic renal failure, dementia, among others); lifestyle and risk factors (eg, obesity, 
hypertension, smoking, alcohol intake, sedentarism); previous treatments (time window: 12 months prior to taking the first anticoagulant) and concomitant treatments (time window: follow-up period). Additionally, information on health services utilisation such as outpatient visits, including medical specialty, hospital admissions and visits to emergency services during the follow-up period and in the previous 12 months, will be collected; (3) healthcare-system-related variables: primary healthcare centre, basic healthcare zone, health department and hospital.

\section{Data analysis plan}

First, the baseline characteristics of the cohort, stratified by VKA and NOACs, will be described. Adherence measures (primary adherence-filling the first prescription -and secondary adherence-PDC and persistence at 3, 6, 12 and 24 months) will be estimated using the appropriate parameters (means or proportions) for each variable with its respective confidence intervals at $95 \%$ (CI 95\%). The monthly PDC of each patient will be used to describe trajectories or patterns of adherence (trajectory models or latent class growth analysis models) for each drug. The selection of the number of trajectory groups or latent classes that best represents the heterogeneity in adherence will be based on: (1) model fit indices: Bayesian information criteria (BIC) ${ }^{70}$ Akaike information criterion (AIC) ${ }^{71}$ where a lower index value indicates a better model fit, and the Lo-Mendell-Rubin likelihood ratio test (LMR-LRT) ${ }^{72}$ which assesses the improvement in fit between neighbouring class models (comparing $\mathrm{k}-1$ and the $\mathrm{k}$ class models). The LMR-LRT test provides a $\mathrm{p}$ value that can be used to determine if there is a statistical improvement in fit for the inclusion of one more class; (2) a minimum proportion of the study sample in a class: 5\%; and (3) entropy, which indicates uncertainty in the classification of the model and is a measure of how well-or how precisely-study participants are classified into their most likely class. The cut-off value will be a posterior probability of $\geq 0.7$.

Later, we will analyse bivariate associations between covariates-patients' characteristics (sociodemographic and clinical), those of the drugs, professionals and healthcare system-and the predefined endpoints (primary and secondary adherence). Multivariate logistic regression analysis will be used to evaluate possible independent associations between covariates of interest (depending on their clinical significance and the results of the previous bivariate analysis), and dichotomous adherence measures (primary adherence and PDC) and multinomial logistic regression models to identify patients' characteristics associated with each trajectory of adherence. The corresponding odds ratios with their respective CIs $95 \%$ will be estimated using backwardforward stepwise methods. The association between the covariates and persistence will be studied with a similar scheme but using Kaplan-Meier models (bivariate analysis) and the Cox proportional hazards regression model (multivariate analysis). The corresponding hazard ratio (HR) will be estimated with their CIs $95 \%$. The predictions from these models will also be used to construct the propensity scores that will be incorporated as explanatory variables with respect to clinical outcomes, in order to minimise confounding.

To analyse the association between different measures of adherence and clinical outcomes, Cox proportional hazard models will be used again, adjusting for the corresponding propensity scores. Cases will be censored at the date of VHA discharge if the main event (hospitalisation for thromboembolic or bleeding events or death) occurs, or at the end of study follow-up. Additionally, in sensitivity analyses, to further assess the robustness of our findings, we will: (1) assess the association between persistence and clinical events by an extension of the Cox models, which allows the use of multiple correlated events, (2) evaluate such association using persistence as a time-varying covariate, (3) evaluate such association using PDC as a time-varying covariate, by calculating PDC in intervals, throughout the length of follow-up. When an event occurs, the PDC for the period preceding such event will be applied. Details on this approach can be seen in $\left(\mathrm{Ho}, 2008\right.$, AHJ) ${ }^{73}$ We will (4) repeat our analysis after excluding days patients spent in the hospital from the denominators of the adherence measures, and (5) when using the PDC as adherence measure, we will also run the models only considering outcomes that occurred at least 12 months after the index date (being the adherence assessment period the first 12 months after the first prescription).

Finally, to estimate the role of different levels of care in the variations of adherence, multilevel logistic or Cox regression models will be used as appropriate, with random effects for the following variables: primary healthcare centre, basic healthcare zone $(n=240)$ and health department $(n=24)$. We will start with the empty model before introducing explanatory variables, including as random effects only the hierarchical levels: individual/ primary healthcare centre/basic healthcare zone/health department. Following the criterion of improvement in the model goodness of fit and parsimony (Deviance Information Criteria) the relevant levels will be chosen, contextual covariates will be incorporated and the interaction between levels will be analysed. Simulation-based Markov Chain Monte Carlo (MCMC) methods will be used to estimate the error terms of the hierarchical structure. Analyses will be performed using STATA and R statistical packages.

\section{Ethics and dissemination}

Interventions derived from the study

This is an observational, retrospective study developed on databases, and does not include any intervention or randomisation, thus it does not imply any additional risk to patients and cannot result in the prescription of any drug. Compliance with the standards of good research practices: This study will be conducted in accordance 
with the international standards for epidemiological studies, as established in the International Guideline for Ethical Review of Epidemiological Studies (Council for the International Organizations of Medical SciencesCIOMS-Geneva, 2009). Clinical Research Ethics Committee. The study protocol was approved by the Clinical Research Ethics Committee of the 'Dirección General de Salud Pública y Centro Superior de Investigación en Salud Pública' (CEIC DGSP-CSISP, ruling of March 5, 2014). Classification and authorisations: The study protocol was submitted to the Spanish Agency of Drugs and Health Products (the competent authority) and classified as a 'postauthorisation study with design other than prospective follow-up' requiring no further authorisations (SPM-ACO-2014-01; 12 March 2014). The study protocol was also authorised by the General Directorate of Pharmacy and Medical Products (23 June 2014) and the cession of anonymised data was approved by the Regulatory Commission of Access to Ambulatory Care Information Access of the VHA. Confidentiality: In accordance with the Spanish laws on right to privacy and patients' protection, and the 2009 and 2012 Resolutions of the Regional Health Secretary regarding information requests from the VHA information system, the database linkages will be carried out in the corresponding Department of the VHA by the people authorised to do these tasks. To protect patient privacy, data will be sent to the researchers with dissociate non-traceable codes that would not allow the identification of individual patients, or their linkage with other databases.

\section{Dissemination plan}

We plan to disseminate the project's findings through peer-reviewed publications and presentations, at relevant national and international health conferences. Policy reports will also be prepared and discussed with stakeholders, in order to promote the translation of our findings into policy and clinical practice. Certain types of measures could be incorporated into the electronic information systems and be incorporated into the sets of performance indicators of the VHA.

\section{EXPECTED LIMITATIONS}

First, the assessment of adherence based on data from the pharmacy electronic records allows a reliable assessment of adherence in terms of prescriptions dispensed. However, there will be no certainty that the patient actually consumes the medication filled from the pharmacy. Nevertheless, studies evaluating the correlation between adherence measures obtained by pharmacy claims and pill counts show a high degree of agreement, ${ }^{74}{ }^{75}$ so high consistency between dispensing and consumption is expected. Second, the misclassification of patients (treated/non-treated or adherent/non-adherent) due to non-registered dispensations, either because they were purchased without a prescription (exceptional for the therapeutic group studied), or because they were acquired in another region (eg, during holiday periods). Third, information biases due to absent registration or differing data recording practices in the electronic medical record. This problem, however, is always present when data from routine clinical practice are used. Fourth, currently, almost all prescriptions in the VHA are issued electronically (less than $1 \%$ of prescriptions are made manually by physicians). However, the study includes data since 2010, when this percentage was slightly higher, although still small (around 5\%). This limitation particularly affects the estimate of primary adherence, because we cannot retrieve those prescriptions issued manually, and in the case of a first prescription not being filled, we would not be able to identify it as non-adherence, thus overestimating real primary adherence. Therefore, for the estimation of primary non-adherence, we will exclude patients not receiving the first prescription through the electronic prescribing module of the electronic medical record due to the inability to establish a prescription-dispensation relationship. Fifth, given the characteristics of the study (observational, based on retrospective real-world data), we can expect the presence of selection bias and confounding, especially in drug selection, because NOAC prescriptions are subject to prior authorisation (requiring a previous failure to keep INR in range using VKA). To address this problem, we have planned the use of matching methods (propensity score).

Funding This study was partially funded by the 2013 Collaboration Agreement between the Fundación para el Fomento de la Investigación Sanitaria y Biomédica (FISABIO) from the Valencia Ministry of Health and Boehringer Ingelheim, a non-conditioned programme to conduct independent research in chronic healthcare, pharmacoepidemiology and medical practice variation. CLR-B was funded by grant RD12/0001/0005 from the Instituto de Salud Carlos III, Spanish Ministry of Health (co-financed by the European Regional Development Fund).

Competing interests SP has received research grants from various pharmaceutical companies and fees for participation in scientific meetings sponsored by pharmaceutical companies. GS-G participated in 2014 in an advisory meeting of Boehringer Ingelheim.

Ethics approval The study protocol was approved by the Clinical Research Ethics Committee of the "Dirección General de Salud Pública y Centro Superior de Investigación en Salud Pública" (CEIC DGSP-CSISP, ruling of March 5, 2014). Classification and authorisations: The study protocol was submitted to the Spanish Agency of Drugs and Health Products (the competent authority) and classified as a 'post-authorisation study with design other than prospective follow-up', requiring no further authorisations (SPM-ACO-2014-01; March 12, 2014). The study protocol was also authorised by the General Directorate of Pharmacy and Medical Products (June 23, 2014) and the cession of anonymised data was approved by the Regulatory Commission of Access to Ambulatory Care Information Access of the VHA.

Provenance and peer review Not commissioned; externally peer reviewed.

Open Access This is an Open Access article distributed in accordance with the Creative Commons Attribution Non Commercial (CC BY-NC 4.0) license, which permits others to distribute, remix, adapt, build upon this work noncommercially, and license their derivative works on different terms, provided the original work is properly cited and the use is non-commercial. See: http:// creativecommons.org/licenses/by-nc/4.0/ 


\section{REFERENCES}

1. Jørgensen HS, Nakayama $H$, Reith J, et al. Acute stroke with atrial fibrillation. The Copenhagen Stroke Study. Stroke 1996;27:1765-9.

2. Lin HJ, Wolf PA, Kelly-Hayes M, et al. Stroke severity in atrial fibrillation. The Framingham Study. Stroke 1996;27:1760-4.

3. Chugh SS, Havmoeller R, Narayanan K, et al. Worldwide epidemiology of atrial fibrillation: a Global Burden of Disease 2010 Study. Circulation 2014;129:837-47.

4. Petersen P, Boysen G, Godtfredsen J, et al. Placebo-controlled, randomised trial of warfarin and aspirin for prevention of thromboembolic complications in chronic atrial fibrillation. The Copenhagen AFASAK study. Lancet 1989;1:175-9.

5. [No authors listed]. The effect of low-dose warfarin on the risk of stroke in patients with nonrheumatic atrial fibrillation. The Boston Area Anticoagulation Trial for Atrial Fibrillation Investigators. N Engl J Med 1990;323:1505-11.

6. [No authors listed]. Stroke Prevention in Atrial Fibrillation Study. Final results. Circulation 1991;84:527-39.

7. [No authors listed]. Warfarin versus aspirin for prevention of thromboembolism in atrial fibrillation: Stroke Prevention in Atrial Fibrillation II Study. Lancet 1994;343:687-91.

8. Lancaster TR, Singer DE, Sheehan MA, et al. The impact of long-term warfarin therapy on quality of life. Evidence from a randomized trial. Boston Area Anticoagulation Trial for Atrial Fibrillation Investigators. Arch Intern Med 1991;151:1944-9.

9. Fuster V, Bhatt DL, Califf RM, et al. Guided antithrombotic therapy: current status and future research direction: report on a National Heart, Lung and Blood Institute working group. Circulation 2012;126:1645-62

10. January CT, Wann LS, Alpert JS, et al. 2014 AHA/ACC/HRS Guideline for the management of patients with atrial fibrillation: a report of the American College of Cardiology/American Heart Association Task Force on Practice Guidelines and the Heart Rhythm Society. J Am Coll Cardiol 2014:pii: S0735-1097(14) 01740-9.

11. Connolly SJ, Ezekowitz MD, Yusuf S, et al. Dabigatran versus warfarin in patients with atrial fibrillation. $N$ Engl J Med 2009;361:1139-51.

12. Patel MR, Mahaffey KW, Garg J, et al. Rivaroxaban versus warfarin in nonvalvular atrial fibrillation. N Engl J Med 2011;365:883-91.

13. Granger CB, Alexander JH, McMurray JJ, et al. Apixaban versus warfarin in patients with atrial fibrillation. $N$ Engl J Med 2011;365:981-92.

14. Miller CS, Grandi SM, Shimony A, et al. Meta-analysis of efficacy and safety of new oral anticoagulants (dabigatran, rivaroxaban, apixaban) versus warfarin in patients with atrial fibrillation. $A m \mathrm{~J}$ Cardiol 2012;110:453-60.

15. Dentali F, Riva N, Crowther M, et al. Efficacy and safety of the nove oral anticoagulants in atrial fibrillation: a systematic review and meta-analysis of the literature. Circulation 2012;126:2381-91.

16. Adam SS, McDuffie JR, Ortel TL, et al. Comparative effectiveness of warfarin and new oral anticoagulants for the management of atrial fibrillation and venous thromboembolism: a systematic review. Ann Intern Med 2012;157:796-807.

17. Bruins Slot KM, Berge $\mathrm{E}$. Factor $\mathrm{Xa}$ inhibitors versus vitamin $\mathrm{K}$ antagonists for preventing cerebral or systemic embolism in patients with atrial fibrillation. Cochrane Database Syst Rev 2013;8: CD008980

18. Ruff CT, Giugliano RP, Braunwald E, et al. Comparison of the efficacy and safety of new oral anticoagulants with warfarin in patients with atrial fibrillation: a meta-analysis of randomised trials. Lancet 2014;383:955-62.

19. Dogliotti A, Paolasso E, Giugliano RP. Current and new oral antithrombotics in non-valvular atrial fibrillation: a network meta-analysis of 79808 patients. Heart 2014;100:396-405.

20. Steinberg BA, Piccini JP. Anticoagulation in atrial fibrillation. BMJ 2014;348:g2116.

21. Lip GY, Larsen TB, Skjøth F, et al. Indirect comparisons of new oral anticoagulant drugs for efficacy and safety when used for stroke prevention in atrial fibrillation. J Am Coll Cardiol 2012;60: 738-46.

22. Schneeweiss S, Gagne JJ, Patrick AR, et al. Comparative efficacy and safety of new oral anticoagulants in patients with atrial fibrillation. Circ Cardiovasc Qual Outcomes 2012;5:480-6.

23. Rasmussen LH, Larsen TB, Graungaard T, et al. Primary and secondary prevention with new oral anticoagulant drugs for stroke prevention in atrial fibrillation: indirect comparison analysis. BMJ 2012;345:e7097.

24. Mantha S, Ansell J. An indirect comparison of dabigatran, rivaroxaban and apixaban for atrial fibrillation. Thromb Haemost 2012;108:476-84.
25. Testa L, Agnifili M, Latini RA, et al. Adjusted indirect comparison of new oral anticoagulants for stroke prevention in atrial fibrillation. QJM 2012;105:949-57.

26. Harenberg J, Marx S, Diener HC, et al. Comparison of efficacy and safety of dabigatran, rivaroxaban and apixaban in patients with atria fibrillation using network meta-analysis. Int Angiol 2012;31:330-9.

27. Assiri A, Al-Majzoub O, Kanaan AO, et al. Mixed treatment comparison meta-analysis of aspirin, warfarin, and new anticoagulants for stroke prevention in patients with nonvalvular atrial fibrillation. Clin Ther 2013;35:967-984.e2.

28. Biondi-Zoccai G, Malavasi V, D'Ascenzo F, et al. Comparative effectiveness of novel oral anticoagulants for atrial fibrillation: evidence from pair-wise and warfarin-controlled network meta-analyses. HSR Proc Intensive Care Cardiovasc Anesth 2013;5:40-54.

29. Sardar P, Chatterjee S, Wu WC, et al. New oral anticoagulants are not superior to warfarin in secondary prevention of stroke or transient ischemic attacks, but lower the risk of intracranial bleeding: insights from a meta-analysis and indirect treatment comparisons. PLOS ONE 2013;8:e77694.

30. Pink J, Pirmohamed M, Hughes DA. Comparative effectiveness of dabigatran, rivaroxaban, apixaban, and warfarin in the management of patients with nonvalvular atrial fibrillation. Clin Pharmacol Ther 2013:94:269-76.

31. Chatterjee S, Sardar P, Biondi-Zoccai G, et al. New oral anticoagulants and the risk of intracranial hemorrhage: traditiona and Bayesian meta-analysis and mixed treatment comparison of randomized trials of new oral anticoagulants in atrial fibrillation. JAMA Neurol 2013;70:1486-90.

32. Skjøth F, Larsen TB, Rasmussen LH, et al. Efficacy and safety of edoxaban in comparison with dabigatran, rivaroxaban and apixaban for stroke prevention in atrial fibrillation. An indirect comparison analysis. Thromb Haemost 2014;111:981-8.

33. Harenberg J, Weiss $\mathrm{C}$. Clinical trials with new oral anticoagulants. Additive value of indirect comparisons also named network meta-analyses. Hamostaseologie 2013;33:62-70.

34. Brown TM, Siu K, Walker D, et al. Development of a conceptual model of adherence to oral anticoagulants to reduce risk of stroke in patients with atrial fibrillation. J Manag Care Pharm 2012;18: 351-62.

35. Glazer NL, Dublin S, Smith NL, et al. Newly detected atrial fibrillation and compliance with antithrombotic guidelines. Arch Intern Med 2007;167:246-52.

36. Broderick JP, Bonomo JB, Kissela BM, et al. Withdrawal of antithrombotic agents and its impact on ischemic stroke occurrence. Stroke 2011;42:2509-14.

37. Deitelzweig SB, Buysman E, Pinsky B, et al. Warfarin use and stroke risk among patients with nonvalvular atrial fibrillation in a large managed care population. Clin Ther 2013;35:1201-10.

38. Bellamy L, Rosencher N, Eriksson B. Adherence to a new oral anticoagulant treatment prescription: dabigatran etexilate. Patient Prefer Adherence 2009;3:173-7.

39. Rodriguez RA, Carrier M, Wells PS. Non-adherence to new oral anticoagulants: a reason for concern during long-term anticoagulation? J Thromb Haemost 2013;11:390-4.

40. Di Minno A, Spadarella G, Tufano A, et al. Ensuring medication adherence with direct oral anticoagulant drugs: lessons from adherence with vitamin $\mathrm{K}$ antagonists (VKAs). Thromb Res 2014;133:699-704

41. Chatterjee S, Sardar P, Giri JS, et al. Treatment discontinuations with new oral agents for long-term anticoagulation: insights from a meta-analysis of 18 randomized trials including 101,801 patients. Mayo Clin Proc 2014;89:896-907.

42. Gallagher AM, Rietbrock S, Plumb J, et al. Initiation and persistence of warfarin or aspirin in patients with chronic atrial fibrillation in general practice: do the appropriate patients receive stroke prophylaxis? J Thromb Haemost 2008;6:1500-6.

43. Fang MC, Go AS, Chang Y, et al. Warfarin discontinuation after starting warfarin for atrial fibrillation. Circ Cardiovasc Qual Outcomes 2010;3:624-31.

44. Glader EL, Sjölander M, Eriksson M, et al. Persistent use of secondary preventive drugs declines rapidly during the first 2 years after stroke. Stroke 2010;41:397-401.

45. Bushnell CD, Olson DM, Zhao $X$, et al. Secondary preventive medication persistence and adherence 1 year after stroke. Neurology 2011;77:1182-90.

46. Rodriguez D, Cox M, Zimmer LO, et al. Similar secondary stroke prevention and medication persistence rates among rural and urban patients. J Rural Health 2011;27:401-8.

47. Gomes T, Mamdani MM, Holbrook AM, et al. Rates of hemorrhage during warfarin therapy for atrial fibrillation. CMAJ 2013;185:E121-7. 
48. Sjölander M, Eriksson M, Glader EL. Few sex differences in the use of drugs for secondary prevention after stroke: a nationwide observational study. Pharmacoepidemiol Drug Saf 2012;21: 911-19.

49. Simons LA, Ortiz M, Germanos $P$, et al. Persistence on warfarin in patients with atrial fibrillation: experience in Australia 2006-2009. Aust Fam Physician 2013:42:659-61.

50. Zalesak M, Siu K, Francis K, et al. Higher persistence in newly diagnosed nonvalvular atrial fibrillation patients treated with dabigatran versus warfarin. Circ Cardiovasc Qual Outcomes 2013;6:567-74.

51. Patel AA, Reardon G, Nelson WW, et al. Persistence of warfarin therapy for residents in long-term care who have atrial fibrillation. Clin Ther 2013;35:1794-804.

52. Tsai K, Erickson SC, Yang J, et al. Adherence, persistence, and switching patterns of dabigatran etexilate. Am J Manag Care 2013;19:e325-32.

53. Schulman S, Shortt B, Robinson M, et al. Adherence to anticoagulant treatment with dabigatran in a real-world setting. J Thromb Haemost 2013;11:1295-9.

54. Shore S, Carey EP, Turakhia MP, et al. Adherence to dabigatran therapy and longitudinal patient outcomes: insights from the veterans health administration. Am Heart J 2014;167: 810-17.

55. Osterberg L, Blaschke T. Adherence to medication. N Engl J Med 2005;353:487-97.

56. Schneeweiss S, Avorn J. A review of uses of health care utilization databases for epidemiologic research on therapeutics. J Clin Epidemiol 2005;58:323-37.

57. Crystal S, Akincigil A, Bilder S, et al. Studying prescription drug use and outcomes with Medicaid claims data: strengths, limitations, and strategies. Med Care 2007;45(10 Supl 2):S58-65.

58. Franklin JM, Shrank WH, Pakes J, et al. Group-based trajectory models: a new approach to classifying and predicting long-term medication adherence. Med Care 2013;51:789-96.

59. Muthén B, Muthén LK. Integrating person-centered and variable-centered analyses: growth mixture modeling with latent trajectory classes. Alcohol Clin Exp Res 2000;24:882-91.

60. Bogner HR, Lin JY, Morales KH. Patterns of early adherence to the antidepressant citalopram among older primary care patients: the prospect study. Int J Psychiatry Med 2006;36: 103-19.
61. Modi AC, Cassedy AE, Quittner AL, et al. Trajectories of adherence to airway clearance therapy for patients with cystic fibrosis. J Pediatr Psychol 2010;35:1028-37.

62. Riegel B. Predictors of objectively measured medication nonadherence in adults with heart failure. Circ Heart Fail 2012;5:430-6.

63. Bastard M, Fall MB, Lanièce I, et al. Revisiting long-term adherence to highly active antiretroviral therapy in Senegal using latent class analysis. J Acquir Immune Defic Syndr 2011;57:55-61.

64. Modi AC, Rausch JR, Glauser TA. Patterns of nonadherence to antiepileptic drug therapy in children with newly diagnosed epilepsy. JAMA 2011;305:1669-76.

65. Ko DT, Chiu M, Guo H, et al. Patterns of use of thienopyridine therapy after percutaneous coronary interventions with drug-eluting stents and bare-metal stents. Am Heart J 2009;158:592-8.e1.

66. Vermeire $\mathrm{E}$, Hearnshaw $\mathrm{H}$, Van Royen $\mathrm{P}$, et al. Patient adherence to treatment: three decades of research. A comprehensive review. $J$ Clin Pharm Ther 2001;26:331-42.

67. Andrade SE, Kahler KH, Frech F, et al. Methods for evaluation of medication adherence and persistence using automated databases. Pharmacoepidemiol Drug Saf 2006;15:565-74.

68. Hess LM, Raebel MA, Conner DA, et al. Measurement of adherence in pharmacy administrative databases: a proposal for standard definitions and preferred measures. Ann Pharmacother 2006;40:1280-8.

69. Sikka R, Xia F, Aubert RE. Estimating medication persistency using administrative claims data. Am J Manag Care 2005;11:449-57.

70. Schwarz G. Estimating the dimension of a model. Ann Stat 1978;6:461-4.

71. Akaike $\mathrm{H}$. A new look at the statistical model identification. IEEE Trans Autom Control 1974;19:716-23.

72. Lo Y, Mendell N, Rubin D. Testing the number of components in a normal mixture. Biometrika 2001;88:767-78.

73. Ho PM, Magid DJ, Shetterly SM, et al. Medication nonadherence is associated with a broad range of adverse outcomes in patients with coronary artery disease. Am Heart J 2008;155:772-9.

74. Steiner JF, Prochazka AV. The assessment of refill compliance using pharmacy records: methods, validity, and applications. J Clin Epidemiol 1997;50:105-16.

75. Grymonpre R, Cheang M, Fraser M, et al. Validity of a prescription claims database to estimate medication adherence in older persons. Med Care 2006;44:471-7. 